
https://doi.org/10.22468/cvia.2016.00143 CVIA 2017;1(1):1-1
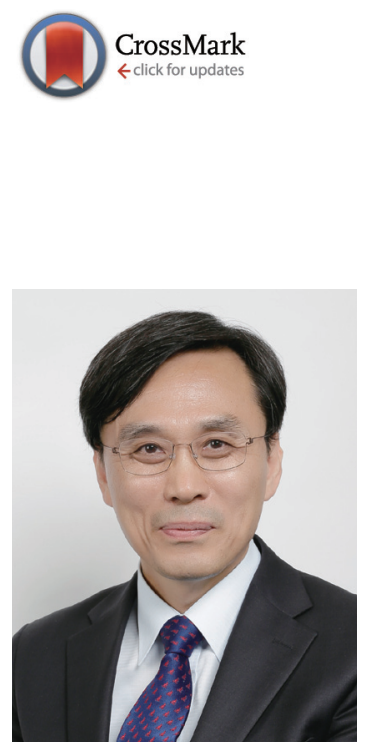

Received: December 20, 2016 Accepted: December 23, 2016

\section{On the Introduction of a New Cardiovascular Imaging Journal in Asia}

\author{
Yeon Hyeon Choe \\ President of Asian Society of Cardiovascular Imaging (ASCI) \\ Department of Radiology, Samsung Medical Center, Sungkyunkwan University \\ School of Medicine, Seoul, Korea
}

It is my great pleasure to announce that Asian Society of Cardiovascular Imaging (ASCI) is launching a new journal on its own platform. It is amazing to see how fast cardiovascular imaging techniques have evolved. This development means that the technologies evoke many questions that must be solved before the techniques can be used as clinical tools. As many clinical researches are necessary as well as an excellent vehicle of education, the birth of new journal is timely for ASCI which has a huge population in the background. It is the hope that Cardiovascular Imaging Asia (CVIA) will provide an excellent medium for the publication of works from not only ASCI researchers, but also those from around the world.

As the first guest Editor-in-Chief and an adjunct editor of the International Journal of Cardiovascular Imaging, I would like to extend my deepest gratitude to Professor Johan H.C. Reiber for his generous support for ASCI special issues from 2009 to 2016. ASCI and its new journal are indebted to Professor Reiber and his journal teams for the current status of the Society. I also appreciate the efforts of Dr. Tae Hoon Kim, Editor-in-Chief, and his task-force team in making this journal visible. Special thanks are given to article authors, editors, and reviewers.

I encourage colleagues devoted to cardiovascular imaging around the world to submit their valuable papers to CVIA. 